Research Article

\title{
Analysing and Data-Driven Modelling the Handling Performance of Rugby Balls under Wet Conditions
}

\author{
Jiaojiao Liu, ${ }^{1}$ Yunan Liu, ${ }^{2}$ Ya Wang, ${ }^{3}$ and Chao Liu $\mathbb{D}^{2}$ \\ ${ }^{1}$ School of Sports and Physical Education, Shandong Sport University, Rizhao, Shandong 276800, China \\ ${ }^{2}$ School of Martial Arts, Shandong Sport University, Rizhao, Shandong 276800, China \\ ${ }^{3}$ Zaozhuang Sport School, Zaozhuang, Shandong 277000, China
}

Correspondence should be addressed to Chao Liu; chaoliu088@gmail.com

Received 24 July 2020; Revised 13 August 2020; Accepted 5 September 2020; Published 14 September 2020

Academic Editor: Jun Chen

Copyright ( $\odot 2020$ Jiaojiao Liu et al. This is an open access article distributed under the Creative Commons Attribution License, which permits unrestricted use, distribution, and reproduction in any medium, provided the original work is properly cited.

This paper studies the effect of moisture on the handling performance of rugby balls, where different moisture conditions will cause a change in skin friction between finger pads and rugby balls. In this study, a set of rugby ball passing tests was performed under wet conditions to test the effect of skin hydration on the performance of ball handling. A comprehensive analysis was then conducted to assess skin frictional properties at various moisture levels. It was observed that skin moisture is strongly associated with the handling performance of rugby balls. Based on the collected experimental data, intelligent data-driven models were finally developed, including a neural network and a fuzzy rule-based system. These models will likely enhance the anticipation of the handling performance of rugby players, which helps in designing specific training programmes and better preparing for rugby games to cope with wet conditions.

\section{Introduction}

As an essential element in most sports games, sports equipment appears to be used very regularly in a variety of forms depending on the sport involved. Examples of the equipment could be balls like basketballs, rugby balls, and footballs, flying discs for freestyle and disc golf, bats for baseball and cricket, rackets for badminton and tennis, etc. In general, this equipment has been designed to offer a high quality of service lives and sports performance with a continuous exploration of new forms of design and materials. For instance, rugby balls were often made by stitching a pig's bladder in an oval shape in early days, and nowadays rugby balls are only allowed to be made of leather or other suitable synthetic materials [1]. In addition, the specification of size, weight, and even the air pressure has been strictly defined according to the rules of the International Rugby Board, with the aim of enhancing the stability of hand gripping [2].

In those sports games involving hands contact with equipment, it is essential for players to demand effective handling skills with the aim of achieving better performance. For example, in rugby, handling skills consist of various activities such as passing balls, catching balls, holding balls, or running with balls. Regardless of position, all players are required to perform those core skills effectively with lower level of errors. This is because ball handling errors are believed intimately associated with the turnover in games. Good ball handling skills could reduce the turnover rate and hence increase the chance of winning [3,4]. Ball handling is a complex activity that employs hands/fingers interacting with ball surfaces under various conditions and can be easily influenced by different factors. For instance, most players find the balls become very slippery when their hands are sweating or they are playing on raining days, which bring difficulties for them to handle the balls with those wet conditions $[5,6]$.

In this paper, we study the handling/passing performance of rugby balls under wet conditions using data analytics and data-driven modelling approaches. In recent years, we have witnessed a dramatic increase in our ability to collect, process, and store data, which also happens in sports 
engineering. Analysing and utilising these data, such as in data-driven modelling, provides humans with very useful knowledge and some practical tools. In the case that some complicated systems cannot be described by conventional mathematical models, people may apply data-driven modelling techniques to develop practical data-driven models. In the current study, we follow this strategy to study the performance of the rugby sport. First, three different experiments were designed and conducted, including a rugby ball passing test, a skin friction test, and a measurement of skin mechanical properties. Data-driven predictive models were then developed based on the experimental data.

\section{Related Work}

In the recent study of Tomlinson et al. [7], they investigated the passing accuracy of various rugby balls under both dry and moist conditions. The coefficient of the friction of the balls was also measured. The results showed that the passing scores of the balls with a lower friction coefficient are poorer than those balls with a higher friction coefficient. Lewis et al. $[8,9]$ pointed that the accuracy of a rugby ball pass is closely associated with the frictional behaviours between human fingers and ball texture. High friction forces would be beneficial for players to handle balls in games. In comparison of balls with various textures, they noticed that the balls with patterns of pimples could help ball gripping under dry conditions. This phenomenon might be attributed to the corresponding change in skin viscoelasticity, which leads the skin to deform around pimples and thus increases the contact area, hence the hysteresis mechanism [7].

The human skin is a complex material and is composed of three different layers. This structure gives the skin unique physical-mechanical properties that are very similar to rubbers $[10,11]$. In past decades, the frictional behaviour of the skin has been studied widely [12-22]. This is because, as the outer covering of the human body, the skin plays an important role in preventing the risk of body damages from skin friction due to the skin contacting with complex environments/objects. It is generally believed that the frictional properties of the skin are owed to various physical mechanisms, for instance, adhesion mechanisms, interfacial shear mechanisms, and capillary action mechanisms. [23]. In addition, it is deemed that skin frictional properties are closely related to the contact conditions: hydration, load, speed, and material properties. For instance, Bowden and Tabor [24] stated that the skin frictional properties comply with a classical two-term model consisting of an adhesion mechanism and a deformation mechanism. In general, it is expected that the coefficient of the skin friction is mainly affiliated with the adhesion mechanism under dry conditions and can be described by a linear relationship [16, 17].

For the moisture conditions, the coefficient of the skin friction is found to vary with different moisture conditions $[13,15,21,22]$. In previous studies, both a linear correlation and a "bell-curve" correlation between the coefficient of skin friction and the moisture level have been observed $[17,22,25,26]$. For example, Yoshimune et al. [25] and Veijgen et al., [26] examined the relationship between the skin moisture level and the coefficient of the skin friction and pointed that the coefficient of skin friction increases with the moisture level following a positive linear function. However, in recent studies, Adams et al. [17] and Tomlinson et al. [22] found a bell-curve relationship for the coefficient of the skin friction when hydrated fingers contacting with various materials, in which an initial increase in the coefficient of friction while adding water to dry skin. It was found that the coefficient of the skin friction decreases after the moisture level of the skin reaches a certain level.

Handling/passing under wet conditions is one of the key research topics in rugby union; however, there is very limited relevant work available. This research was performed with the aim of investigating how the handling/passing performance is influenced by different moisture levels using data analytics and data-driven modelling techniques. In this study, three different experiments were designed and conducted, including a rugby ball passing test, a skin friction test, and a measurement of skin mechanical properties. Predictive models were then developed based on the experimental data.

\section{Experiments}

3.1. Rugby Ball Passing Test under Different Moisture Conditions. In this test, eight male rugby players (age $20 \pm 2$ years old) from Shandong Sport University took part, and they all have developed similar ball passing/handling skills. The details of all participants were recorded, including height, weight, wrist strength, throwing posture, and handling hand preference. Prior to the test, all participants were given a training session to familiarise the test procedure. In order to investigate the effect of the moisture level on the accuracy of ball handling, the participants were asked to perform the test under four different moisture conditions: a natural state, a medium hydration state, a high hydration state, and an addition of water. A moisture meter was used to measure the moisture level of the skin on the participants' thumb and middle fingers of their right hands (see Liu et al. [19] for more details).

As illustrated in Figure 1, the participants were instructed to run with a rugby ball from position $\mathrm{E}$ to zone $\mathrm{AB}$ (the distance between $\mathrm{E}$ and $\mathrm{AB}$ is $4 \mathrm{~m}$ ) and throw the ball to a target net when arrived at zone $\mathrm{AB}$. The target net was located at a place $7 \mathrm{~m}$ away from $\mathrm{AB}$. Then, the participants needed to run toward position $\mathrm{F}$ to pick another ball and back to zone AB to complete another shot. Prior the tests, all participants were required to clean their hands and dry them with paper towels. Regarding the natural-state test, it was performed on cleaned and dried hands. For the middle hydration test, the participants were requested to carry out $10 \mathrm{~min}$ warm-up activities firstly and then perform the passing test immediately in order to minimise the loss of water from the skin. In the third test of a relative high hydration state, the participants were asked to perform the passing test along 20 min warm-up activities for a higher moisture level. Finally, the participants were asked to perform the test using wet balls. In order to ensure all surfaces of the balls are covered with water, the balls were soaked in 


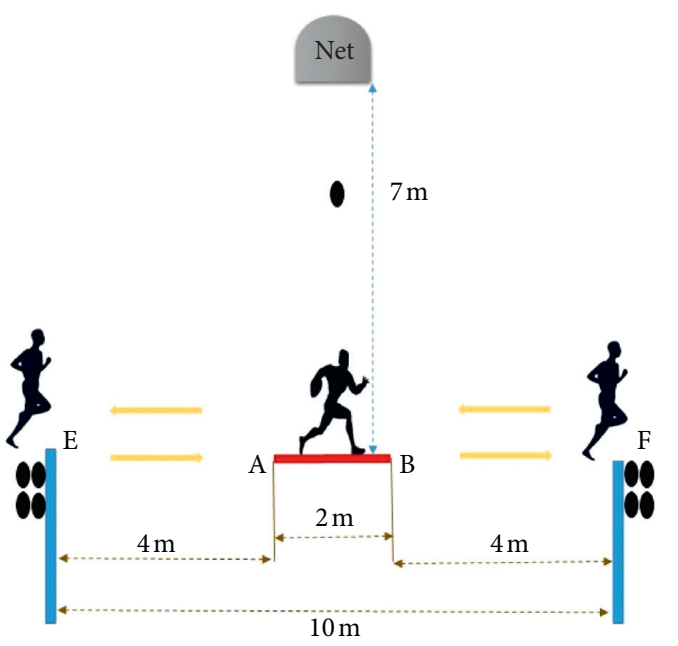

(a)

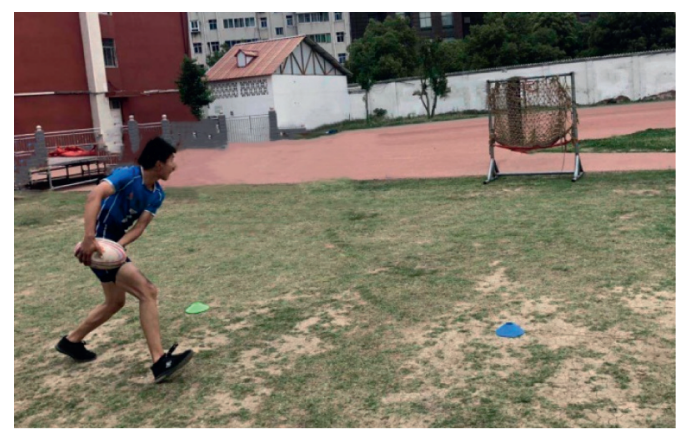

(b)

Figure 1: (a) Sketch of the passing ball test and (b) images of passing a ball to the net.

water for $1 \mathrm{~min}$ before the test. With regard to each moisture condition, the participants had to complete one successful shot. The time taken for completing all shots successfully by each participant was recorded for determining their final passing scores. The scoring scheme used in this study is given in Table 1 [27]. During the test, if any invalid run or invalid data were identified, the test would be repeated until getting the valid data. After the passing test, 32 experimental data were collected.

All obtained data from the rugby ball passing test were entered into Microsoft Excel and analysed by the SPSS software (version 26) using a nonparametric method (Friedman test) and a correlation analysis method. Prior to these inferential statistical analyses, all raw data need to be preprocessed. In this study, the moisture readings were processed for the average data and standard deviations using a descriptive analysis. Due to the big varieties of the moisture readings collected from participants, it is necessary to normalise the data for each player. The normalised moisture readings were set in the range of 0.0 to 1.0. The Friedman test was employed to analyse the difference in the moisture readings among four hydration conditions. In addition, the relationship between the normalised moisture readings and the target scores was also studied by correlation analyses. In all statistical analyses, the $p$ value that is less than 0.05 was deemed to be statistically significant. The correlation coefficient $R$ was also calculated to analyse the relationship between the measured data and the predicted results.

3.2. Measurement of Skin Friction. A miniature force platform device (Mode: HE6X6, Advanced Mechanical Technology, Inc.) was employed to measure skin friction. It is a low-load, high-accuracy measurement device $(22 \mathrm{~N}$ in $X$ and $Y$-axes and $44 \mathrm{~N}$ in $Z$-axis with an accuracy of $1 \%)$ and consists of a force plate, an interface box, and a computer (see [18] for more details). The principle of this measuring device relies on the strain gauge flexibility technique that allows the forces to be detected in $X$-, $Y$-, and $Z$-axes. During measurement of friction coefficient, the normal force implied to the force acting in the $Z$-axis and the corresponding force in $X-/ Y$-axis is considered as a friction force; hence, the coefficient of skin friction can be obtained by the proportion of the friction force to the normal force. In the current study, the measurements of the coefficient of the skin friction were performed on the middle fingers of the participants. The participants were instructed to slide the examined fingers along a $5 \mathrm{~mm}$ wide acetal strip (roughness of $0.5 \mu \mathrm{m}$ ) with a normal force of $1.5 \pm 0.2 \mathrm{~N}$. Prior the tests, all participants were invited and requested to clean and dry their hands. With regard to a variety of moisture levels in the skin, the participants were asked to soak their right hands in tap water for $20,40,80,120,160,200,240,280,320,360$, and $400 \mathrm{~s}$. The moisture level of the skin was recorded by a moisture meter. All friction measurements were repeated three times to obtain average results.

3.3. Measurement of Skin Mechanical Properties. This series of tests aimed to explore the mechanical properties of fingerpad skin in response to skin hydration. To achieve this, the middle fingers on the right hands of participants were examined using a noninvasive Cutometer MPA 580 (see [19] for more details). The participants were asked to prepare the examined fingers using the same method of the skin friction measurement. The test firstly was performed with a dry finger and then a soaked finger ( $400 \mathrm{~s}$ of soaking time). With the purpose of avoiding the influence of any chemical on measurements, no treatment was given to the examined finger in twelve hours before the test. A $2 \mathrm{~mm}$ diameter probe was held on the surface of the examined finger with a constant pressure of $500 \mathrm{mbar}$ at a room temperature. The measurements were repeated three times to ensure the accuracy of the results. 


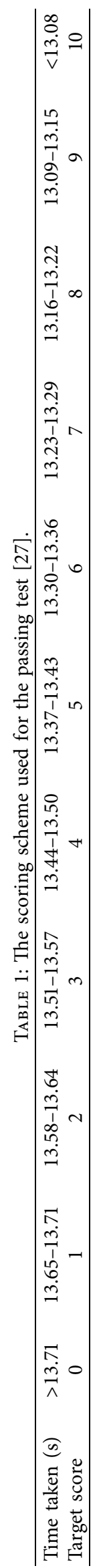




\section{Data Analysis and Discussion}

4.1. Rugby Ball Passing Test. As shown in Table 2, the moisture readings rise significantly from the "natural" state to the "hydrated" state when the participants were involved in warm-up activities. This finding is also evaluated by the Friedman test where big differences on the moisture level of the skin were obtained under four moisture conditions $(p<0.001)$. It can be observed that the moisture readings of the examined fingers reach their maximum levels (99 au) in the case of doing $20 \mathrm{~m}$ warm-up activities. With regard to the condition of extra water, the tests were performed by saturated fingers with wet balls where additional water was added to rugby ball surfaces, prior to the tests. For the target score, a different phenomenon was observed with respect to various moisture conditions. An initial increase in the target score was found when the moisture level changing from the natural state to the Hydrated 1 state. After that, the target score started to decrease with the increase in moisture readings. The lowest score was found in the case of adding extra water, as given in Table 2. These findings suggest a parabolic relationship of the target score with the moisture level (see Figure 2). A quadratic polynomial model was considered to provide the best fit for these changes in the target score and expressed as $y=a x^{2}+b x+c(R>0.9$, $p<0.03)$.

Figure 2 shows the data for the passing test under four moisture conditions. For the moisture reading, it was found to be varied with warm-up activities. For 10 min exercise, the moisture reading has a significant increase with time. As shown in Figure 2, the moisture reading for Participant 1 in the natural condition was $69 \mathrm{au}$ and increased to $73 \mathrm{au}$ after taking part in $10 \mathrm{~min}$ of warm-up. A similar finding was also noted by Tomlinson et al. [22], in which the moisture levels of the tested fingers were found to increase from $50 \mathrm{au}$ in resting to $90 \mathrm{au}$ when the participants were taking part in various activates. Their findings agree with this study. The changes in the moisture level of the skin could be attributed to the sweating on the skin surface. During various activities, participants' bodies experience high temperature as a result of body cells converting metabolic energy into thermal energy. In order to maintain the balance of temperature, excessive heat generated is converted into thermoregulatory sweating and evaporated through the skin. Consequently, the sweating accumulated on the skin surface leads to a change in the skin moisture.

The data of the target score with respect to different moisture conditions are also given in Figure 2, where a bellcurve relationship between the target score and the moisture level is observed. The target score is found to have an initial increase with the increase in moisture and then a decrease when the moisture level reaches Hydration 1, except Participant 7. In Participant 7, the target score for Hydrated 2 shows an increasing phenomenon with the increase in the moisture level. This could be attributed by many factors, such as participant's skill, test conditions, and others. The bell-curve behaviour in the ball pass test was expected because of the curved relationship between skin friction and moisture of the skin. Lewis et al. $[8,9]$ indicated that the accuracy of a ball pass is closely associated with the frictional behaviour between the finger pad and ball texture. It is generally believed that a high friction force is desired for a good grip and therefore a higher accuracy of pass rate. In addition, they indicated the coefficient of the skin friction is inversely proportional to the skin's Young's modulus. An appropriate increase in the moisture level of the skin could reduce its Young's modulus and, therefore, result in a rise in the coefficient of the skin friction. In the studies of Tomlinson et al. [7], they found the target score changes with the skin friction coefficient following a linear regression model. In the comparison of the skin friction coefficient between the dry balls and the wet balls, the results show that the wet balls have relative lower coefficients than those of dry balls. Moreover, in their passing accuracy test, the balls with higher friction coefficient give better scores than those with lower friction coefficient.

On the basis of the above findings, it is suggested that the dry balls with a relative low moisture level will present a good friction coefficient and, therefore, a good passing accuracy. Oppositely, wet balls usually give a lower friction coefficient and a lower passing accuracy. This conclusion is in consonance with the findings of this study. When the participants were in activities (up to $10 \mathrm{~min}$ ), the moisture level was found to increase due to thermoregulatory sweating and this leads to an increase in the target score. While increasing the warm-up time, the mechanical properties of the skin could be affected due to excessive sweating and consequently result in a decrease in skin friction and then a lower target score. With regard to the extra water condition, the lowest target score is obtained. This could be attributed to that the additional water on the ball surface acts as a lubricant at the contact interface. The water-based lubrication film produced a lower friction between the hand and the ball surface and led to a lower target score.

4.2. Skin Friction. A curve relationship was observed between the moisture reading and the hydration time, as shown in Figure 3(a). It was noticed that the moisture reading is increased by $55 \%$ with hydration. In the natural state, the moisture reading was 53 au and rose to 82 au after being hydrated for $80 \mathrm{~s}$. After that, the moisture reading showed a decreasing tendency with the hydration time and reached a plateau around $200 \mathrm{~s}$. Figure 3(b) shows the data of the skin friction coefficient collected from different moisture conditions. It indicated that the coefficient of the skin friction varies with hydration time with an initial increase in the coefficient of the skin friction when the examined fingers were hydrated for around $80-120 \mathrm{~s}$. Then, it is followed by an approximately $35 \%$ of decrease in the coefficient of the skin friction with time. This finding conforms to the work of Tomlinson et al. [22]. In their studies, an initial increase in the coefficient of friction was observed with a small amount of water added to participants' hands, and then this was followed by a decrease. Additionally, in some other studies, Adams et al. [17] carried out a work on the frictional behaviour of the human skin where a polypropylene probe was sliding on participant's forearms under different moisture 
TABLE 2: The results collected from the target passing test.

\begin{tabular}{|c|c|c|c|c|c|}
\hline \multirow{2}{*}{\multicolumn{2}{|c|}{ Participants }} & \multicolumn{4}{|c|}{ Moisture levels } \\
\hline & & Natural & $\begin{array}{c}\text { Hydrated } \\
1\end{array}$ & $\begin{array}{c}\text { Hydrated } \\
2\end{array}$ & $\begin{array}{l}\text { Extra } \\
\text { water }\end{array}$ \\
\hline \multirow[t]{2}{*}{1} & $\begin{array}{l}\mathrm{MMR} \pm \mathrm{SD} \\
(\mathrm{au})\end{array}$ & $71.3 \pm 1.8$ & $73.0 \pm 1.8$ & $96.0 \pm 2.8$ & $99.0 \pm 0.0$ \\
\hline & Target score & 6 & 9 & 6 & 5 \\
\hline \multirow[t]{2}{*}{2} & $\begin{array}{l}\mathrm{MMR} \pm \mathrm{SD} \\
(\mathrm{au})\end{array}$ & $56.3 \pm 1.3$ & $72.3 \pm 1.0$ & $99.0 \pm 0.0$ & $99.0 \pm 0.0$ \\
\hline & Target score & 5 & 7 & 5 & 4 \\
\hline \multirow[t]{2}{*}{3} & $\begin{array}{l}\mathrm{MMR} \pm \mathrm{SD} \\
\quad(\mathrm{au})\end{array}$ & $51.3 \pm 2.8$ & $69.7 \pm 1.8$ & $86.7 \pm 6.9$ & $99.0 \pm 0.0$ \\
\hline & Target score & 7 & 8 & 8 & 5 \\
\hline \multirow[t]{2}{*}{4} & $\begin{array}{c}\mathrm{MMR} \pm \mathrm{SD} \\
(\mathrm{au})\end{array}$ & $56.0 \pm 4.4$ & $77.0 \pm 3.6$ & $99.0 \pm 0.0$ & $99.0 \pm 0.0$ \\
\hline & Target score & 7 & 9 & 7 & 7 \\
\hline \multirow[t]{2}{*}{5} & $\begin{array}{l}\mathrm{MMR} \pm \mathrm{SD} \\
(\mathrm{au})\end{array}$ & $67.0 \pm 1.6$ & $75.0 \pm 2.8$ & $99.0 \pm 0.0$ & $99.0 \pm 0.0$ \\
\hline & Target score & 6 & 8 & 6 & 6 \\
\hline \multirow[t]{2}{*}{6} & $\begin{array}{l}\mathrm{MMR} \pm \mathrm{SD} \\
(\mathrm{au})\end{array}$ & $68.0 \pm 1.7$ & $72.0 \pm 0.0$ & $99.0 \pm 0.0$ & $99.0 \pm 0.0$ \\
\hline & Target score & 7 & 8 & 5 & 4 \\
\hline \multirow[t]{2}{*}{7} & $\begin{array}{l}\mathrm{MMR} \pm \mathrm{SD} \\
(\mathrm{au})\end{array}$ & $62.7 \pm 4.3$ & $71.3 \pm 0.8$ & $93.3 \pm 3.9$ & $99.0 \pm 0.0$ \\
\hline & Target score & 5 & 7 & 8 & 5 \\
\hline \multirow[t]{2}{*}{8} & $\begin{array}{l}\mathrm{MMR} \pm \mathrm{SD} \\
(\mathrm{au})\end{array}$ & $66.7 \pm 0.8$ & $78.0 \pm 0.4$ & $99.0 \pm 0.0$ & $99.0 \pm 0.0$ \\
\hline & Target score & 7 & 9 & 6 & 6 \\
\hline
\end{tabular}

Note. MMR represents a mean moisture reading collected from both examined fingers, Hydrated 1 represents a medium hydration state, and Hydrated 2 represents a high hydration state.

conditions. The coefficient of the skin friction obtained was found to be $0.2 \mu$ for the dry skin and rose to $4.2 \mu$ after adding demineralised water to the examined skin. The friction coefficient then dropped to $0.2 \mu$ when water was removed. Based on those observations, they suggested that the coefficient of the skin friction and the hydration time are in consonance with a curve relationship (bell-shaped).

The changes in the coefficient of the skin friction under moisture conditions might be ascribed to the growth of the contact area between the skin and the force probe/plate. When adding water to the interface, some water would be absorbed by skin tissues, which will help soften the skin and lead to an increment in the contact area. According to Amontons laws, the coefficient of the skin friction is proportional to the contact area; thus, a rise in the coefficient of the skin friction can be expected due to the increased contact area. For the water remaining on the skin surface, it develops into "liquid bridges" at the interface. Those liquid bridges formed might introduce viscous shear stress into the contact and therefore lead to increases in the contact area and the coefficient of the skin friction. The capillary adhesion might also be found to increase, which is attributed to the increased contact area.

4.3. Mechanical Properties of the Skin. The results of the mechanical properties under hydration are shown in
Figure 4. It is noted that the skin deformation is reduced from $0.14 \mathrm{~mm}$ to $0.11 \mathrm{~mm}$ with the hydration time of $120 \mathrm{~s}$. After that, it is found to gradually recede to $0.11 \mathrm{~mm}$. An opposite phenomenon was found in the global elasticity of the skin $\left(U_{r} / U_{e}\right.$, where $U_{r}$ is the immediate retraction and $U_{e}$ is the immediate distension). Figure 4(b) shows that the global elasticity reaches to 0.7 with the hydration time of $120 \mathrm{~s}$ and then decreases to a steady value of 0.5 at $400 \mathrm{~s}$. The viscoelasticity $\left(U_{v} / U_{e}\right.$, where $U_{v}$ is the delayed distension) was found to be strongly associated with the hydration time as there is a significant reduction observed in Figure 4(c). A curved behaviour was also noted in the normal stiffness with the hydration time, as shown in Figure 4(d). Similar to that of the global elasticity, the normal stiffness has an initial increase of $35 \%$ at $120 \mathrm{~s}$, followed by a decline to 0.17 at $400 \mathrm{~s}$.

In previous studies of the skin's mechanical properties, Yuan and Verma [28] indicated that the elastic modulus of the stratum corneum (SC) can be obtained using a noninvasive technique by using a Hysitron TriboScope. The elastic modulus can be defined as a function of unloading stiffness:

$$
E=\frac{\sqrt{\pi}}{2 \sqrt{A}} *\left(\frac{\mathrm{d} N}{\mathrm{~d} L}\right)
$$

where $E$ is Young's modulus, $A$ is the contact area, $N$ is the normal load applied on the skin surface, and $L$ is the skin deformation subject to the normal load [28]. In their comparative study of elastic modulus in the dry and wet skin, the results show that the elastic modulus of the dry skin is one order of magnitude higher than that of the wet skin. Hendricks and Franklin [15] found that the skin stiffness for finger pads is decreased by about an order of magnitude while increasing its moisture level. The results of Liu et al.'s studies indicated that the elastic modulus and the normal stiffness start to decrease while increasing the thicknesses of the artificial fingers [19]. Their further studies of rubbing tests indicated that one of the possibilities for the reduction in friction force is likely to be the change in SC thickness. According to Liu et al. [19], the adhesion force is in inverse proportion to skin stiffness. The removal of SC does not affect the normal stiffness of the skin, but it does affect the lateral stiffness of the skin. This finding is in good accordance with our observation in Figure 4(d) as no obvious change was noted in the normal stiffness of the skin. In a similar study carried out by Pailler-Mattei et al. [21], they pointed out that the lateral stiffness of the skin is decreased with the removal of the thickness of the SC. They assumed that the change in lateral stiffness might influence the global mechanical properties of the skin and hence influence its friction coefficient.

From the above findings, it is concluded that the coefficient of the skin friction closely correlates with the SC thickness. Therefore, it is expected to observe a decrease in the elastic modulus of the skin while increasing the thickness of the skin related to hydration. Figure 4(a) shows that there is an initial decrease and then an increase in the deformation when the finger pads were hydrated in water up to $400 \mathrm{~s}$. However, the corresponding change in the global elasticity of the skin is not significant. One of the possible causes could 

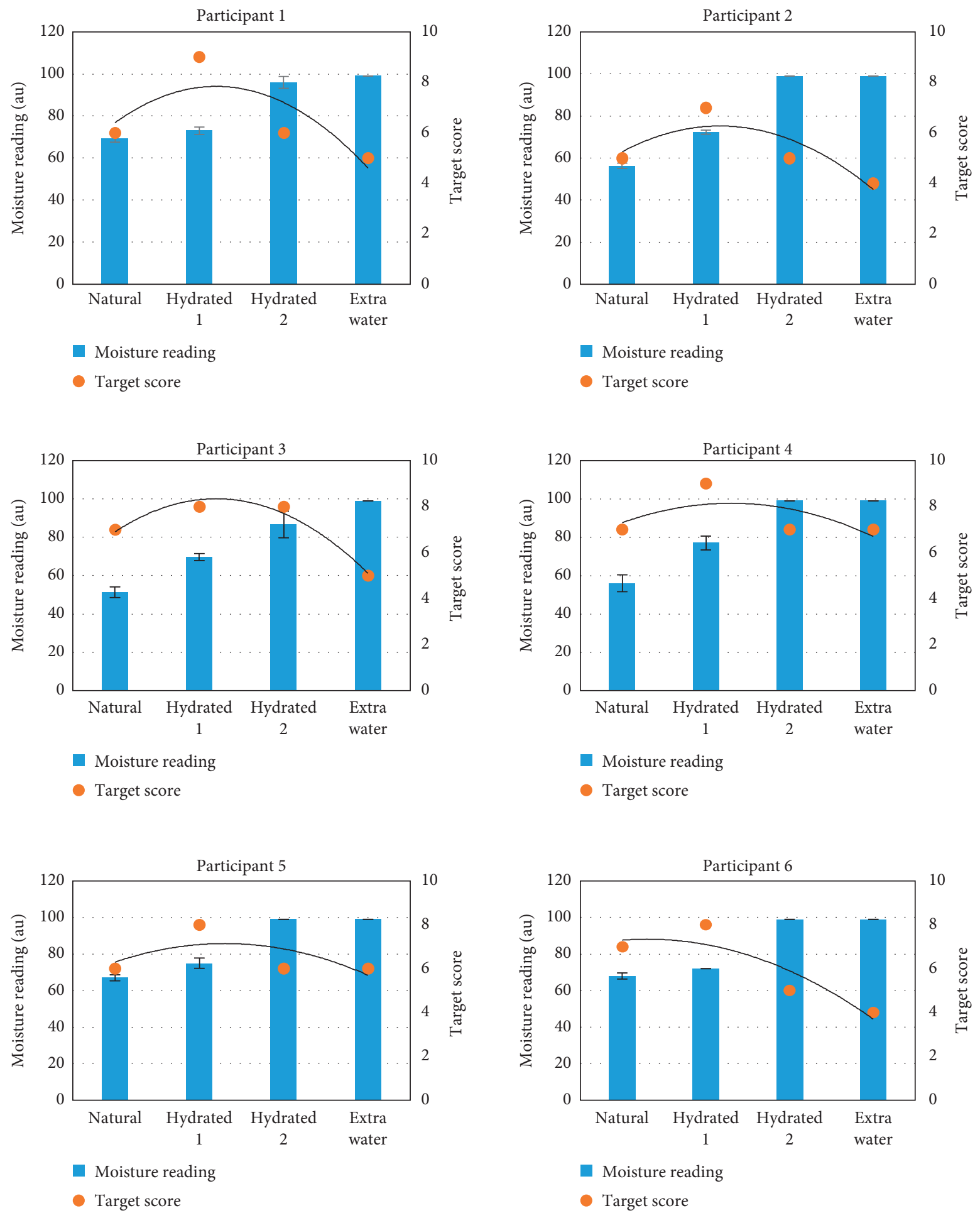

(a) 

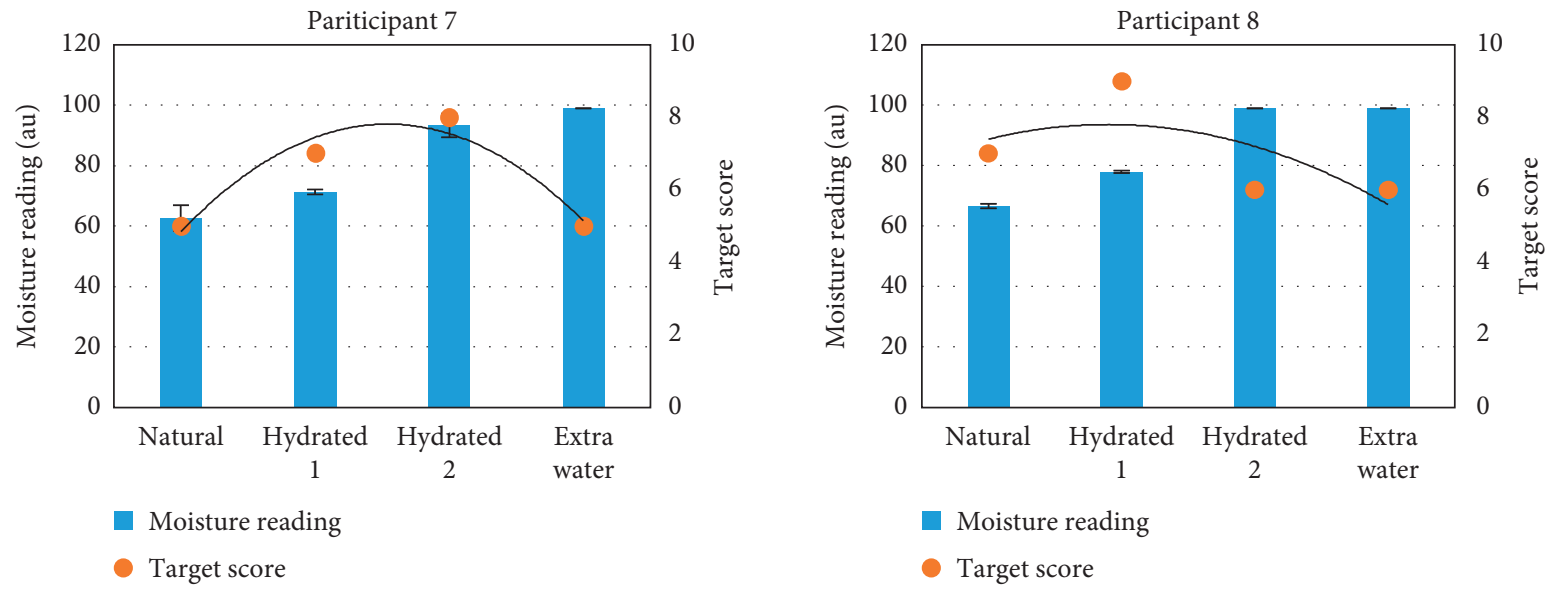

(b)

FIGURE 2: The moisture readings and the target scores under different hydration conditions.

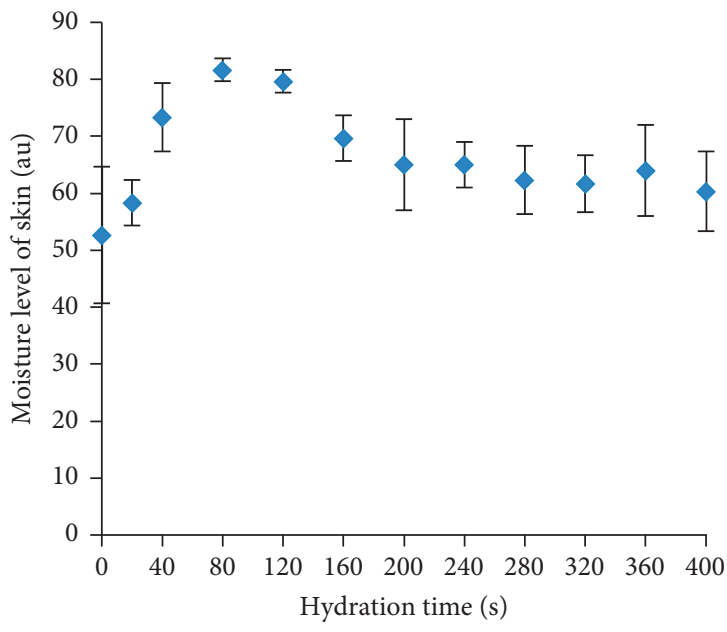

(a)

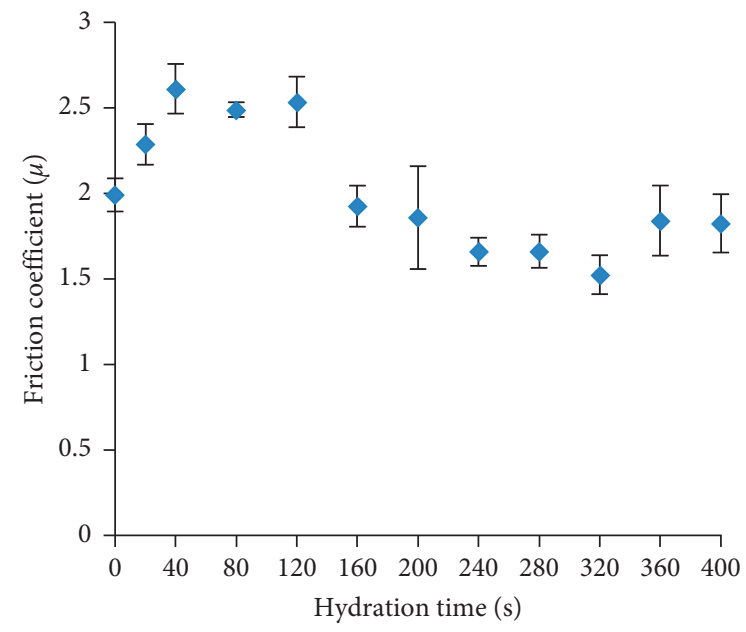

(b)

Figure 3: (a) The moisture reading versus the hydration time and (b) the skin friction coefficient versus the hydration time.

be that data of the elasticity obtained are of the global elasticity for the whole skin. The global elasticity is believed to be dominated by the dermis layer of the skin, so it might be not very accurate to describe the elastic property of the SC. In addition, in the studies of Liu et al. [27], they indicated that no water is transmitted into the dermis layer of the skin during the hydration treatment. Therefore, it is reasonable to observe that the elasticity of the skin does not have significant changes under wet conditions.

\section{Data-Driven Models}

To generate predictive models for anticipating the passing scores of athletes, two widely used data-driven modelling techniques were employed. The first one is a feedforward artificial neural network (ANN) with a Levenberg-Marquardt training method [29]. In this paper, the ANN model was set to have a hidden layer with eight sigmoid neurons. The second method used is the adaptive neuro-fuzzy inference system (ANFIS) [30]. It is a Sugeno fuzzy rule-based system that uses the back-propagation gradient descent and least-squares methods in training. In this paper, the ANFIS model constructed has eight fuzzy rules.

In the experiments, six variables were selected as the inputs of models, which are athletes' weight, height, hand length, hand width, wrist strength, and figure moisture level. The output of models is the score of the passing test. The figure moisture level has been observed to be correlated with the ball handling performance in Section 4. The weight and height represent the athletes' physical conditions and often reflect the strength and speed of athletes. The hand length, hand width, and wrist strength directly relate to the ball handling performance of humans. These variables are believed to be associated with the capability of passing rugby 


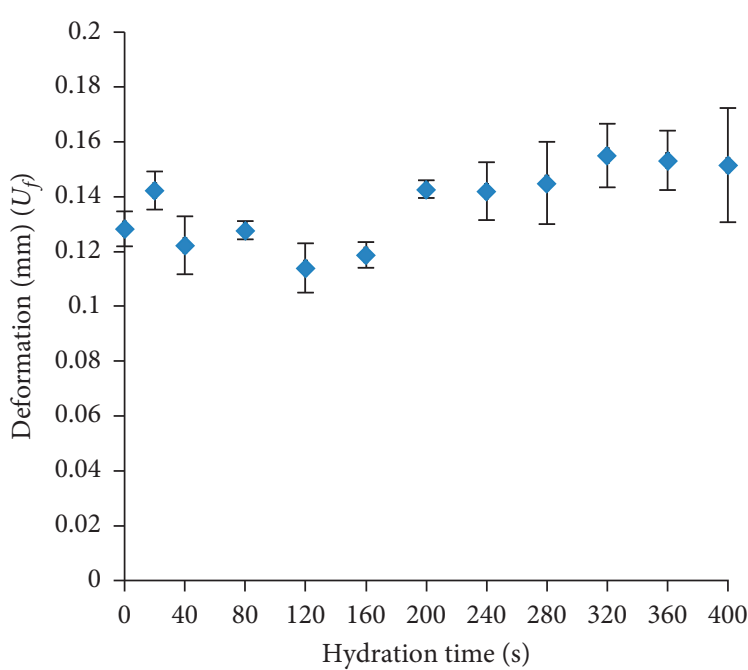

(a)

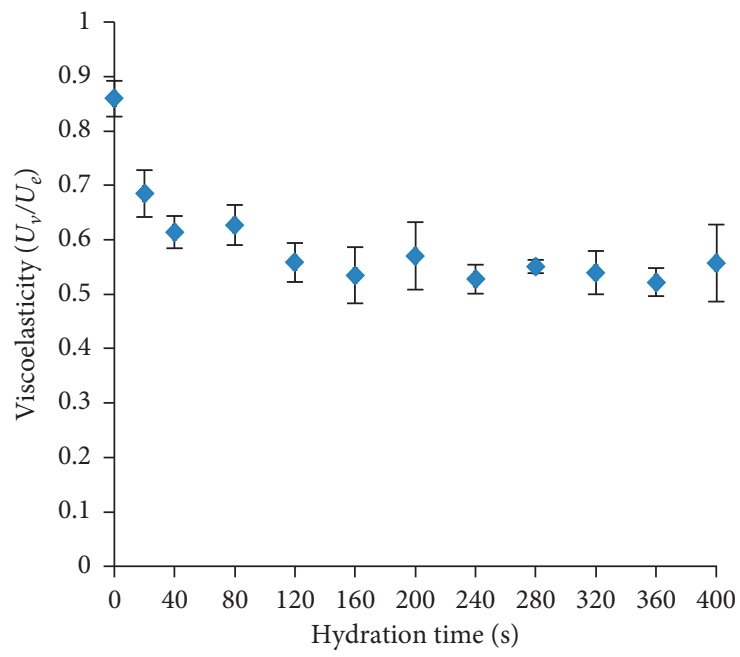

(c)

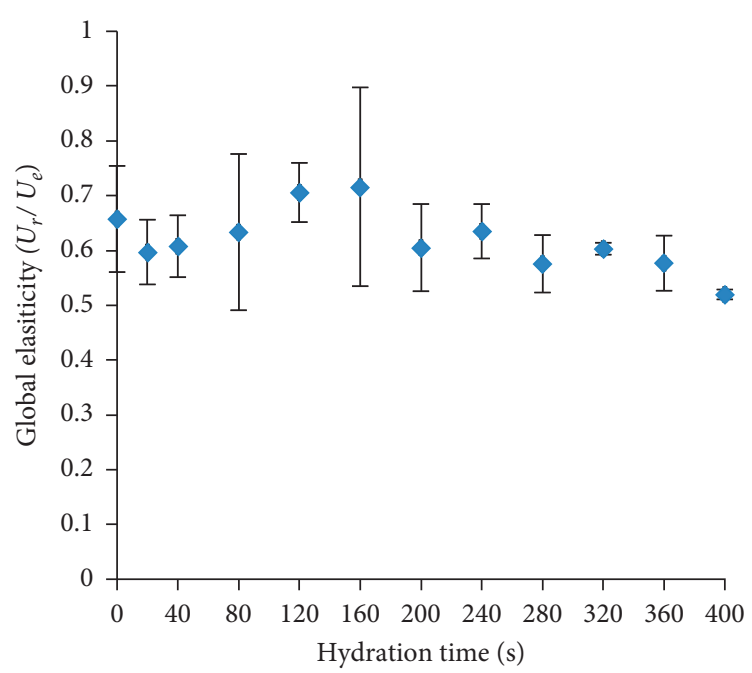

(b)

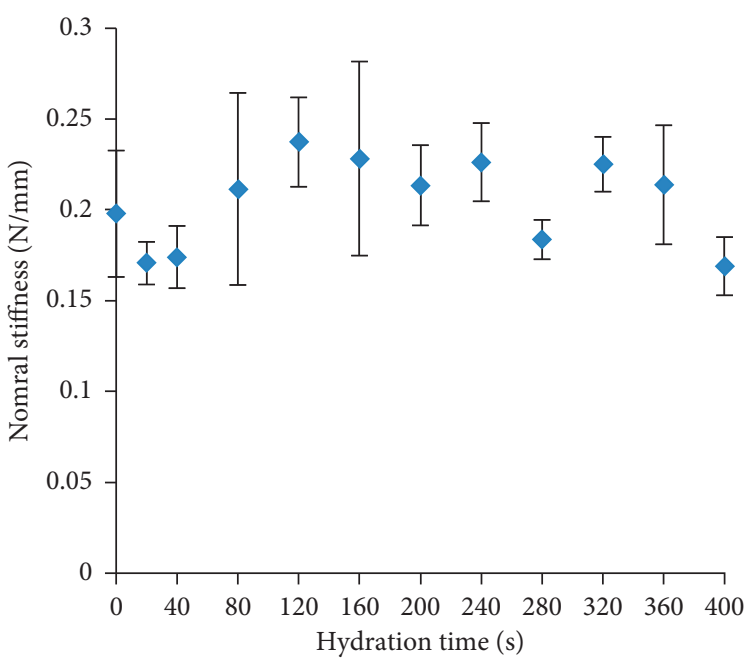

(d)

FIgUre 4: (a) Skin deformation $\left(U_{f}\right)$ against hydration time, (b) global elasticity $\left(U_{r} / U_{e}\right)$ against hydration time, (c) viscoelasticity $\left(U_{v} / U_{e}\right)$ against hydration time, and (d) normal stiffness against hydration time.

TABLE 3: Basic information about athletes.

\begin{tabular}{lccccc}
\hline Participants & Weight $(\mathrm{kg})$ & Height $(\mathrm{m})$ & Hand length $(\mathrm{cm})$ & Hand width $(\mathrm{cm})$ & Wrist strength $(\mathrm{N})$ \\
\hline 1 & 73 & 1.77 & 19 & 20 & 20 \\
2 & 85 & 1.8 & 19 & 21 & 80 \\
3 & 61 & 1.78 & 18 & 19 & 60 \\
4 & 80 & 1.8 & 22 & 19 & 80 \\
5 & 75 & 1.8 & 18 & 19 & 50 \\
6 & 89 & 1.85 & 18 & 20 & 50 \\
7 & 64 & 1.76 & 18 & 20 & 60 \\
8 & 62 & & 1.7 & 19 & \\
\hline
\end{tabular}

balls and thus are used as input variables of models. Table 3 shows the basic information about athletes, which was used in modelling. In the experiments, $80 \%$ of the collected data were randomly selected and used in training and the remaining $20 \%$ of the data were utilised in testing. Table 4 and Figure 5 show the performance of the developed models in prediction. In Table 4, RMSE represents root mean square error and $R$ represents Pearson product-moment correlation coefficient.

It can be observed that both the ANN and ANFIS models perform well in the training data, but they are less accurate in testing. Compared with the ANFIS model, the ANN model is less accurate in training; however, it performs much better in testing. The reason lies in that ANN has a validation 
TABLE 4: Modelling performance using ANN and ANFIS.

\begin{tabular}{llccr}
\hline Model & Training & \multicolumn{2}{c}{ Testing } & RMSE \\
\hline ANN & RMSE & $R$ & 0.6158 & 0.9916 \\
ANFIS & 0.2663 & 0.9841 & 7.6267 & 0.8853 \\
\hline
\end{tabular}

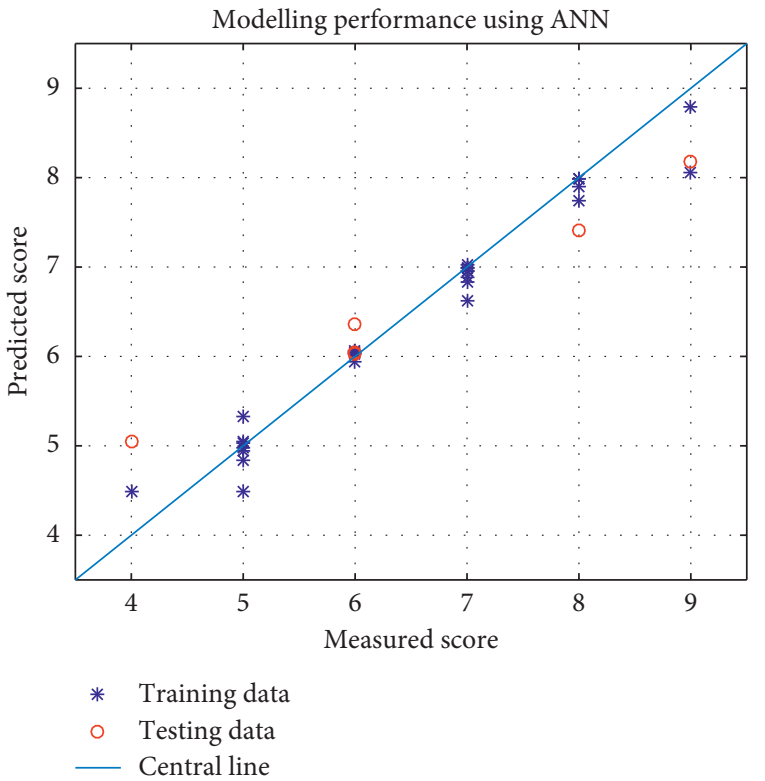

(a)

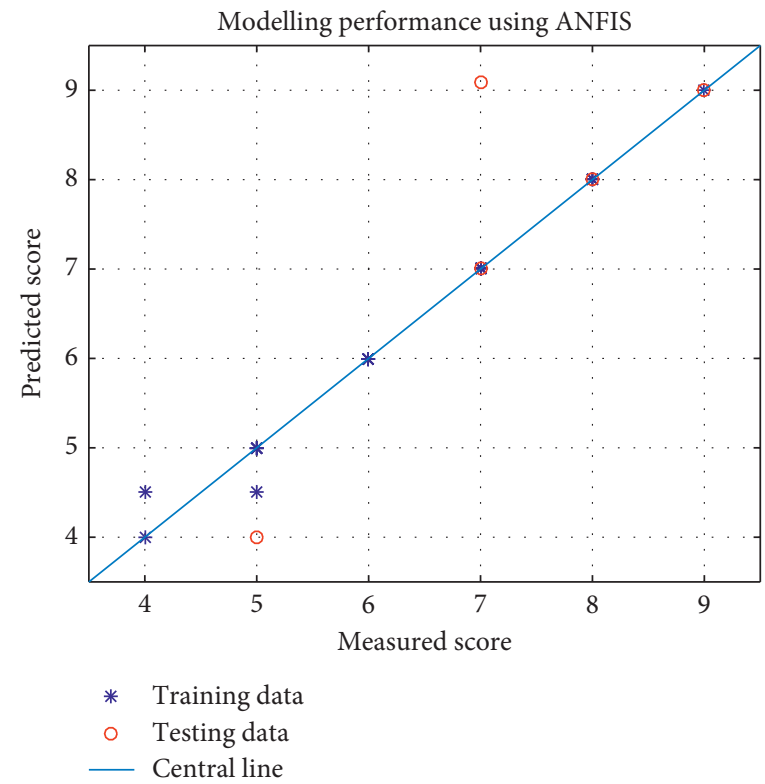

(b)

Figure 5: Measured passing score versus predicted passing score using (a) ANN and (b) ANFIS.

mechanism that can prevent overtraining, which happened in the training of the ANFIS model.

\section{Conclusion}

The aim of this study was to investigate rugby players' performance under different moisture conditions using data analytics and data-driven modelling methods. In the rugby ball passing test, it has been found that higher target scores can be obtained by increasing the skin moisture to a certain level. When the finger pads were saturated, the passing accuracy started to drop. A quadratic polynomial function has been proposed to describe the relationship between the skin moisture and the target score. The measurement of the skin friction coefficient showed that there is bell-shaped behaviour between the coefficient of the skin friction and the hydration time. Additionally, in the measurement of mechanical properties, the skin elasticity of the finger pads was shown to reduce with hydration. Based on the collected experimental data, intelligent data-driven models have then been developed to anticipate the passing performance of rugby players under different moisture conditions, by employing a neural-network model and a neuro-fuzzy model. The constructed models showed decent accuracy, although with limited training data.

\section{Data Availability}

The data used in this research are available from the corresponding author upon request.

\section{Conflicts of Interest}

The authors declare that they have no conflicts of interest.

\section{References}

[1] "Pioneers of the rugby ball," 2019, http://www. rugbyfootballhistory.com/ball.htm.

[2] Board International Rugby, Law 2 the Ball, Playing Charter, International Rugby Board, Dublin, Ireland, 2006.

[3] A. Ross, "Physical characteristics and match performance in rugby sevens," Auckland University of Technology Theses and Dissertations Archive, Auckland, New Zealand, 2015, https:// openrepository.aut.ac.nz/bitstream/handle/10292/9215/RossA. pdf?sequence $=1$, Doctoral dissertation.

[4] L. Vaz, S. Hendricks, and W. Kraak, "Statistical review and match analysis of Rugby World Cups finals," Journal of Human Kinetics, vol. 66, no. 1, pp. 247-256, 2019.

[5] A. Woolford, "Why player jerseys are being blamed for so many people dropping the ball at the Rugby World Cup," 2019, https://www.walesonline.co.uk/sport/rugby/rugbynews/player-jerseys-being-blamed-many-17029726. 
[6] P. Williams, "Five things we learnt about rugby in September," Rugby World, 2019, https://www.rugbyworld.com/ tournaments/rugby-world-cup-2019/five-things-learntrugbyseptember-2-103598.

[7] S. E. Tomlinson, R. Lewis, S. Ball, A. Yoxall, and M. J. Carré, "Understanding the effect of finger-ball friction on the handling performance of rugby balls," Sports Engineering, vol. 11, no. 3, pp. 109-118, 2009.

[8] R. Lewis, M. J. Carré, A. Abu Bakar, and S. E. Tomlinson, "Effect of surface texture, moisture and wear on handling of rugby balls," Tribology International, vol. 63, pp. 196-203, 2013.

[9] R. Lewis, M. J. Carré, and S. E. Tomlinson, "Skin friction at the interface between hands and sports equipment," Procedia Engineering, vol. 72, pp. 611-617, 2014.

[10] A. Delalleau, G. Josse, J.-M. Lagarde, H. Zahouani, and J.-M. Bergheau, "A nonlinear elastic behavior to identify the mechanical parameters of human skin in vivo," Skin Research and Technology, vol. 14, no. 2, pp. 152-164, 2008.

[11] S. Derler, M. Preiswerk, G.-M. Rotaru, J.-P. Kaiser, and R. M. Rossi, "Friction mechanisms and abrasion of the human finger pad in contact with rough surfaces," Tribology International, vol. 89, pp. 119-127, 2015.

[12] O. S. Dinc, C. M. Ettles, S. J. Calabrese, and H. A. Scarton, "Some parameters affecting tactile friction," Journal of Tribology, vol. 113, no. 3, pp. 512-517, 1991.

[13] L.-C. Gerhardt, V. Strässle, A. Lenz, N. D. Spencer, and S. Derler, "Influence of epidermal hydration on the friction of human skin against textiles," Journal of the Royal Society Interface, vol. 5, no. 28, pp. 1317-1328, 2008.

[14] F. M. Hendriks, "Mechanical behaviour of human epidermal and dermal layers in vivo: characterization of non-linear mechanical behaviour of skin using ultrasound," Technische Universiteit Theses and Dissertations Archive, Eindhoven, Netherlands, Doctoral dissertation, 2005.

[15] C. P. Hendriks and S. E. Franklin, "Influence of surface roughness, material and climate conditions on the friction of human skin," Tribology Letters, vol. 37, no. 2, pp. 361-373, 2010.

[16] S. A. Johnson, D. M. Gorman, M. J. Adams, and B. J. Briscoe, "The friction and lubrication of human stratum corneum," "The friction and lubrication of human stratum corneum," in Thin Films in Tribology, D. Dowson, C. M. Taylor, T. H. C. Childs, M. Godet, and G. Dalmaz, Eds., Elsevier Science Publishers, Amsterdam, Netherlands, pp. 663-672, 1993.

[17] M. J. Adams, B. J. Briscoe, and S. A. Johnson, "Friction and lubrication of human skin," Tribology Letters, vol. 26, no. 3, pp. 239-253, 2007.

[18] X. Liu, Z. Lu, R. Lewis, M. J. Carré, and S. J. Matcher, "Feasibility of using optical coherence tomography to study the influence of skin structure on finger friction," Tribology International, vol. 63, pp. 34-44, 2013.

[19] X. Liu, D. Gad, Z. Lu, R. Lewis, M. Carré, and S. Matcher, "The contributions of skin structural properties to the friction of human finger-pads," Proceedings of the Institution of $\mathrm{Me}$ chanical Engineers, Part J: Journal of Engineering Tribology, vol. 229, no. 3, pp. 294-311, 2015.

[20] X. Liu, M. J. Carré, Q. Zhang, Z. Lu, S. J. Matcher, and R. Lewis, "Measuring contact area in a sliding human fingerpad contact," Skin Research and Technology, vol. 24, no. 1, pp. 31-44, 2018.

[21] C. Pailler-Mattei, S. Pavan, R. Vargiolu, F. Pirot, F. Falson, and H. Zahouani, "Contribution of stratum corneum in determining bio-tribological properties of the human skin," Wear, vol. 263, no. 7-12, pp. 1038-1043, 2007.

[22] S. E. Tomlinson, R. Lewis, X. Liu, C. Texier, and M. J. Carré, "Understanding the friction mechanisms between the human finger and flat contacting surfaces in moist conditions," Tribology Letters, vol. 41, no. 1, pp. 283-294, 2011.

[23] S. E. Tomlinson, R. Lewis, and M. J. Carré, "Review of the frictional properties of finger-object contact when gripping," Proceedings of the Institution of Mechanical Engineers, Part J: Journal of Engineering Tribology, vol. 221, no. 8, pp. 841-850, 2007.

[24] F. P. Bowden and D. Tabor, The Friction and Lubrication of Solids, Clarendon Press, Oxford, UK, 1954, https://scholar. google.com/scholar_lookup?title=The $\% 20$ Friction $\% 20$ and $\%$ 20Lubrication\%20of\%20Solids\&publication year=1954\&author=Bowden\%2CFP\&author=Tabor\%2CD

[25] N. Yoshimune, F. Takaharu, A. Yuichiro et al., "Tactile impression and friction of water on human skin," Colloids and Surfaces B: Biominterfaces, vol. 69, no. 2, pp. 264-267, 2009.

[26] N. K. Veijgen, M. A. Masen, and E. van der Heide, "A novel approach to measuring the frictional behaviour of human skin in vivo," Tribology International, vol. 54, pp. 38-41, 2012.

[27] J. Liu, Q. Zhang, and X. Liu, "Understanding handling performance of rugby balls under wet conditions: analysis of finger-ball friction," International Journal of Performance Analysis in Sport, vol. 20, no. 5, pp. 782-799, 2020.

[28] Y. Yuan and R. Verma, "Measuring microelastic properties of stratum corneum," Colloids and Surfaces B: Biointerfaces, vol. 48, no. 1, pp. 6-12, 2006.

[29] F. D. Foresee and M. T. Hagan, "Gauss-Newton approximation to Bayesian learning," in Proceedings of International Conference on Neural Networks (ICNN'97), pp. 1930-1935, Houston, TX, USA, June 1997.

[30] J.-S. R. Jang, "ANFIS: adaptive-network-based fuzzy inference system," IEEE Transactions on Systems, Man, and Cybernetics, vol. 23, no. 3, pp. 665-685, 1993. 